\title{
Improving patient safety by implementing a new general surgical on-call rota for FY1 doctors
}

Henry Walton, Mokthar Uheba

Royal Sussex County Hospital, Brighton, UK

\begin{abstract}
Junior doctors often feel they have little control over their working environment and can feel overwhelmed by the numerous barriers to their involvement with rota design. This project aimed to overcome these barriers to change by implementing a new FY1 on-call rota, designed by junior doctors, for the surgical department at the Royal Sussex County Hospital, Brighton.
\end{abstract}

A new rota was necessary since the department had recently undergone an organisational restructuring that altered the out of hours work load for FY1 doctors. To address this, the proposed new rota increased FY1 presence during weekend daytime shifts.

To assess junior opinion about the old rota and the proposed new rota, a survey was sent to $32 \mathrm{FY} 1$ doctors analysing their views about each rota in terms of safety, the sufficiency of cover for various shifts, and their willingness to work the rota. A direct comparison between the two rotas was also requested. A statistically significant $(p<0.05, n=12$, two-tailed paired T-test) increase in people agreeing with the following statements about the new rota: "The rota is safe", "This rota has sufficient FY1 cover during the weekend day shifts", and "I would be prepared to work this rota." A direct comparison revealed that FY1 doctors felt that the new rota would be safer, they would be more willing to work the new rota, and that the new rota should be implemented. Implementation occurred for the August 2014 general surgical FY1 intake and post implementation audits showed that the new FY1s felt that cover across all shifts was improved and that the new rota was safe.

This project demonstrates that altering rotas can improve junior doctor's perception of patient safety, and also shows that junior team members, with departmental support, can overcome barriers to change and implement new rotas.

\section{Problem}

The department of surgery at the Royal Sussex County Hospital, UK recently underwent organisational changes including a major alteration of team structures and instituting a consultant-led ward round every day of the week for all general surgical inpatients. These changes were not accompanied by a new junior on-call rota and the foundation year one doctors (FY1) in particular noted that they had a significant increase in their weekend workload due to the ward round. As a result, the department wanted to implement a new surgical FY1 on-call rota which would address this issue. The department also wanted to ensure that the new rota improved patient safety and would improve morale among the FY1 team.

\section{Background}

There is currently a debate within the medical community about the advantages of introducing a seven day working week to help mitigate a reported increase in mortality rates at weekends.[6] Recently both the Royal College of Physicians [7] and Royal College of Surgeons [9] have voiced their support for a seven day working week, with the Royal College of Physicians detailing how this may be achieved in their "Future Hospital Commission" report.[8] These discussions must therefore be considered when designing new rotas as it appears likely that all hospitals will soon be required to introduce a seven day working week.
Junior doctors can often feel that they have little control over their working environment [2], especially since on-call rotas are often designed by non-clinical staff who clinical staff rarely meet. Furthermore, altering out of hours shift patterns can result in alterations to pay supplements that can be difficult to gain approval for. Additionally, the regular rotation of junior doctors through a department also means that we rarely stay long enough to benefit from the changes we initiate. These factors mean that junior doctors are not frequently involved in designing their own rotas, which appears to be a lost opportunity since not only are junior doctors individually affected by rota design but they are also in a unique position to detect flaws in the rota and provide creative solutions. Their involvement is especially important since rotas can often have an impact on both individual stress and patient safety.[1, 2, 3]

\section{Baseline measurement}

A survey was sent to $32 \mathrm{FY} 1$ doctors (with 15 responding) who had worked within the department under the old rota asking them to rate six statements about the "old" rota from 1 - 5 ( 1 = strongly disagree, $3=$ neither agree nor disagree and $5=$ strongly agree). The mean score for each statement was then calculated. The six statements focused on assessing the FY1 views on the safety of the rota, the sufficiency of cover for various shifts and their willingness to work this rota.

Most FY1s strongly disagreed or disagreed with the statement "This 
rota is safe" (mean = 2.27). A similar result was seen with the statement "This rota has sufficient FY1 cover during the weekend day shifts" (mean $=1.67$ ). This was in comparison to neutral or positive feelings about cover during weekday day shifts, night shifts, and twilight shifts (see table 1 for full baseline results).

See supplementary file: ds4971.pptx - "Table 1"

\section{Design}

These responses about the "old" rota showed that FY1 doctors were concerned about its safety, with a particular cause of concern being the level of cover during the weekend day shift. The "proposed new rota" sought to address these concerns by scheduling a third FY1 doctor to be on-call during the weekend day shift and keeping the levels of staffing during the other out-of-hours shifts the same. FY1 doctors were allocated three extra lieu days across the four month rotation to compensate for working an extra weekend.

\section{Strategy}

PDSA cycle 1: Initially we surveyed 32 FY1 doctors and asked them to rate six statements (discussed above) for both of the "old" and "proposed new" rotas. We also asked them to directly compare the two rotas with regard to three statements and gave them the opportunity to leave written comments about the rotas. Overall the results (discussed in detail in results section) showed broad support for the rota changes.

Comments from the initial survey indicated that there was consensus that the pay banding should be increased to $50 \%$ (from $40 \%$ ) to reflect the increase in out-of-hours work, therefore this was implemented (subject to human resources (HR) approval).

Comments also suggested that FY1 doctors were routinely working beyond their contracted hours due to the volume of work within the department. As this appeared to be impacting team morale, the new rota was modified so that a standard day finished half an hour later at 17:30, thus reflecting realistic working patterns. Two extra lieu days across a four month rotation were inserted into the rota in order to compensate for this increase in hours.

This modified rota was sent to the trust's HR department who analysed the rota to ensure it was compliant with the European Working Time Directive (EWTD).[4] Although they agreed that it was EWTD compliant, they were concerned that small amounts of overtime would quickly cause the doctors to become non-EWTD compliant, so they requested that two further lieu days be inserted into the rota. This was duly done. They also confirmed an increase in pay banding to $50 \%$.

The rota was then sent for departmental approval. This was granted and implemented for the August 2014 intake of FY1 doctors.

PDSA cycle 2: The August 2014 intake of FY1 doctors were resurveyed by asking them to rate the same original six statements about the new rota and provide comments (see results). The results suggested an improvement in patient safety and comments were broadly positive; no further alterations to the rota were deemed necessary at this point.

PDSA cycle 3: The December 2014 intake of FY1 doctors were resurveyed in the same manner (see results), and these results suggested less satisfaction with the weekend daytime cover than the previous cohorts working the new rota. This was addressed by hiring a locum doctor in the morning on weekends with the sole responsibility for writing discharge summaries. Comments further indicated that there were concerns that bank holidays were not staffed as well as the weekend day shifts. There was also confusion about how doctors were allocated between the vascular and urology teams with a lack of definition, leading to certain teams being overstaffed and other teams being understaffed. This feedback was relayed to the rota coordinator who has ensured that more people are present on bank holidays for the next rotation. The department has also defined on the rota who is covering vascular and urology to ensure that the work force is evenly distributed between the two teams.

Due to a national shift in policy with a greater focus on community care [10], the local deanery has decided to move FY1 posts from surgical departments to community posts. The general surgical department will be over-hauling all of their rotas to adjust to changes in their levels of staffing. As a result this is the last rota in this format produced for the FY1s and so no further audit is proposed.

\section{Results}

When collecting the baseline data, the FY1 doctors were also asked to rate the new rota for the same six statements (12 out of 15 respondents continued to this section of the survey). The mean scores showed a large improvement for the statements "the rota is safe" (mean from 2.27 to 3.62) and "this rota has sufficient cover during weekend day shifts" (mean from 1.67 to 3.93 ). Full results can be found in table 1 .

To assess if these changes were significant, a paired, two tailed Ttest was performed using the twelve surveys which responded to questions about both the old and the new rota. There was a statistically significant $(p<0.05, n=12)$ increase in people agreeing with the following statements about the new rota: "The rota is safe" ( $T=4.22)$, "This rota has sufficient FY1 cover during the weekend day shifts" ( $T=6.07)$, and "I would be prepared to work this rota" $(\mathrm{T}=2.65)$.

These twelve FY1 doctors were also asked to directly compare the two rotas. These results showed that they felt that the new rota would be safer $(83.33 \%$ vs $0 \% ; 16.67 \%$ stated rotas equal), they would prefer to work the new rota ( $75 \%$ vs $8.33 \% ; 16.67$ stated rotas equal) and the new rota should be implemented ( $91.67 \%$ vs $8.33 \%)$.

After the rota had been implemented with a few minor alterations (as discussed in strategy and improvement cycles section) we then 
BMJ Quality Improvement Reports

re-audited ten new FY1 doctors. They rated the same six statements about the new rota that the previous departmental FY1s had responded to. The new FY1s felt that the new rota was safe (mean score $=3.80$ ) and displayed similar levels of support for the new rota that the previous FY1s had displayed (see table 2 for complete results). Notably the new FY1s responded positively to all six statements.

For the second rotation that this rota was used, we also re-audited the FY1s in the same manner. These FY1s felt that the new rota was safe (mean score $=3.50$ ) and displayed similar levels of support for the new rota that previous FY1s had displayed (see table 2 for complete results). The exception to this is that they felt that weekend daytime cover was not sufficient (mean score $=2.40$ ).

\section{Lessons and limitations}

The success of this project largely rested on the multidisciplinary teamwork involved in creating the new rota. Gaining support from senior consultants increased the likelihood of approval for the new rota. Gaining support from the surgical service manager meant that we received sufficient funding for the rota changes and helped to obtain approval from HR for an EWTD compliant rota. As an FY1 doctor, the primary author's role was designing and auditing this new rota, thus ensuring that the rota was based on evidence from junior doctors about their working patterns and that the junior doctors were willing to work the new rota. Trying to implement the new rota without this multidisciplinary team approach would not have been possible.

This project uses FY1 perception of patient safety as an indicator of patient safety within the department. Although this is a subjective measure of patient safety, more objective measures were not suitable since implementation of the new rota coincided with the August change over of doctors, a period which has previously been reported to have an increased patient mortality rate of 6\%.[5] Any alteration in objective measures, such as the number of reported safety incidents within the department, may therefore be attributable to staffing changes rather than alterations to the rota.

This audit is limited by small sample sizes which were unavoidable since only $32 \mathrm{FY} 1$ doctors had worked under the old rota and only $18 \mathrm{FY} 1$ doctors had worked under the new rota in each rotation. Response rates were however relatively good ( $38 \%$ for initial survey and $56 \%$ for each re-audit); despite these small sample sizes, we still had statistically significant results when undertaking the paired T-test comparison of results.

At the time the initial survey was conducted, the primary author was an FY1 doctor within the department and his familiarity with the survey respondents may have affected the results. However, his role within the department meant he was in a unique position to design the survey and seek others opinions. To mitigate this bias, an online format was used when sending the surveys to colleagues and neutral language was used when sending e-mails to request survey completion.

\section{Conclusion}

General surgical FY1 doctors felt that the old rota had insufficient FY1 cover during weekend day shifts and that this was impacting on patient safety. Implementing this new rota has addressed this imbalance and will aim to improve patient safety within the department. Its implementation has been supported at a junior level, as evidenced by the fact the majority of FY1s would prefer to work the new rota. It has also been supported at a senior level, as evidenced by the fact that the rota has been approved and adopted by the department. The new surgical FY1 doctors working the new rota support it and feel that the new rota is safe and has improved the level of cover across all shifts. The perception of patient safety within the department has therefore been improved due to the implementation of this new rota.

In summary, this project demonstrates that altering rotas can improve junior doctor's perception of patient safety. It also shows that junior team members can implement new rotas by gaining senior and administrative support and providing evidence that the change will be beneficial for patients and the department.

\section{References}

1. Reid W. "European Working Time Directive legislation: Developing and implementing organisational practice that delivers better, safer care". Qual Saf Health Care 2004;13:4 247-8.

2. Firth-Cozens $\mathrm{J}$, Cording $\mathrm{H}$. "What matters more in patient care? Giving doctors shorter hours of work or a good night's sleep?" Qual Saf Health Care 2004;13:165-6.

3. Firth-Cozens J, Greenhalgh J. "Doctors' perceptions of links between stress and lowered clinical care". Soc Sci Med 1997;44:1017-22.

4. Temple J. "Time for Training: A Review of the impact of the European Working Time Directive on the quality of training" Conference of postgraduate medical deans of the UK.

5. Jen M, Bottle A, Majeed A, Bell D and Aylin P. "Early InHospital Mortality following Trainee Doctors' First Day at Work". PLoS One 2009 4(9):e7103.

6. Bell C and Redelmeier, D. "Mortality among patients admitted to hospitals on weekends as compared with weekdays". New Engl J Med 2001;345(9):663-8.

7. Royal College of Physicians. "Position statement: Care of medical patients out of hours" 2010.

https://www.rcplondon.ac.uk/sites/default/files/rcp-positionstatement-care-of-medical-patients-out-of-hours.pdf

8. Royal College of Physicians. "Future Hospital: Caring for Medical Patients". 2013.

https://www.rcplondon.ac.uk/sites/default/files/futurehospital-commission-report.pdf

9. Royal College of Surgeons. "Comment on BBC Radio 4 programme on seven day care in hospitals". 2013. http://www.rcseng.ac.uk/news/comment-on-bbc-radio-4-pro gramme-on-seven-day-care-in-hospitals\#.VCWybPldWuo

10. UK Foundation Programme Office. "The UK Foundation Programme Reference Guide". 2012 (modified 2014) 


\section{Declaration of interests}

Data included in this submission was presented as a poster and an oral presentation at the DAPS Global Summit in Nottingham on 14th November 2014.

\section{Acknowledgements}

Mrs Patsy Huckle, surgical services manager. 